\title{
The Acute Effect of Repeated Sprints on Inter-limb Asymmetries during Unilateral Jumping
}

\begin{abstract}
The aim of the present study was to investigate the effects of multiple repeated sprints on unilateral jump performance and inter-limb asymmetries. Eighteen recreationally active males performed three single leg countermovement jumps (SLCMJ) as baseline data. The repeated sprint protocol was $6 \times 40$ m with 20 seconds of passive rest between each sprint. This protocol was conducted four times, each set separated by four minutes of rest. Within that rest period, subjects performed one SLCMJ on each limb after two minutes of rest. A one-way ANOVA showed significant reductions $(p<0.05$; ES $=-0.52$ to -0.99 ) in jump height on both limbs after each set relative to baseline. Inter-limb asymmetries increased at each time point and ranged from 7.62-14.67\%, with significant increases in asymmetry seen after sets three $(p=0.046)$ and four $(p=0.002)$. Significant increases in sprint time were shown between sprints one and six in each set $(p \leq 0.01)$. A fatigue index $(\%)$ was also calculated and showed an exponential increase from $5.74 \%$ (set one) to $13.50 \%$ (set four), with significant differences between all sets $(p<0.001)$ with the exception of sets three and four. Results from this study show that a 6 x $40 \mathrm{~m}$ repeated sprint protocol is a sufficient dose for implementing acute fatigue in recreationally active subjects. This was manifested by reductions in jump height at all time points and jump height asymmetries after the third and fourth sets. These findings indicate that jump height from unilateral jump testing may be a useful metric to use during the monitoring process in recreationally trained athletes.
\end{abstract}

Key Words: Symmetry, fatigue, sprint decrement, countermovement jump 
Effects of Repeated Sprints on Asymmetries

\section{INTRODUCTION}

Inter-limb asymmetry is defined as the difference in function or performance of two limbs (6). Asymmetries have been shown to be present in athletes across a range of sporting activities, including soccer (32), swimming (16), basketball (33) and sprinting (17). They have been shown to be a product of sporting engagement (23), and even a necessity for safe and effective participation for some athletes (21). Further to this, inter-limb asymmetries have been observed across a range of physical characteristics such as strength $(1,2,22)$, power $(4,19)$, and leg stiffness (29). Recent studies have examined the effects of between-limb asymmetries on measures of physical performance. Lockie et al. (27) and Dos Santos et al. (15) showed that heightened jump height and hop distance asymmetries respectively, had no effect on speed and change of direction (CODS) performance. Conversely, Maloney et al. (29) showed that unilateral drop jump height asymmetries were associated with slower CODS times $(r=0.6 ; p$ $=0.026$ ) in recreational athletes. Furthermore, Bishop et al. (7) showed that single leg countermovement jump (SLCMJ) height asymmetries were associated with slower $5 \mathrm{~m}(r=$ $0.49 ; p<0.05), 10 \mathrm{~m}(r=0.52 ; p<0.05)$ and $20 \mathrm{~m}(r=0.59 ; p<0.01)$ sprint performance. Considering the potential reductions in physical performance associated with inter-limb asymmetries, evaluating potential methods to test for their prevalence is warranted.

Single leg jump testing is frequently used to quantify asymmetries as unilateral movements are commonplace in a range of sports and these assessments are simple and time-efficient $(8,24,27,29)$. This method can also be used as a means to detect neuromuscular fatigue $(12,34)$. Cormack et al. (14) showed that multiple metrics (including jump height) can be computed reliably when athletes perform single and repeated countermovement jumps. More recently, Bishop et al. (9) highlighted a distinct lack of literature on how fatigue effects asymmetry with the majority of studies in this area examining inter-limb differences during running tasks. 
Effects of Repeated Sprints on Asymmetries

Radzak et al. (30) measured kinetic and kinematic asymmetries during a fatigue-inducing treadmill protocol and showed significant between-limb differences in 8 out of 32 variables during running. Girard et al. (19) examined the effects of 5x5-second treadmill sprints on kinetic and kinematic running asymmetries. Kinetic asymmetries ranged from $4.1-12.8 \%$ whilst kinematic asymmetries ranged from 1.6-9.0\%, but none of the changes in asymmetries reached statistical significance. However, it is questionable whether the subjects achieved a substantial degree of fatigue, given the small sprint decrement value of $3.1 \%$ across all sprints, compared to values of between $3.53 \%$ and $7.72 \%$ being defined as a suitable fatigue decrement in previous research (20).

In contrast, there is a paucity of research examining the acute effects of fatigue on asymmetry measured during jumping tests. Bromley et al. (10) investigated the use of the SLCMJ as a means of monitoring changes in jump performance and inter-limb asymmetries at incremental time points after a competitive soccer match. Results showed that jump performance was reduced and between-limb differences significantly increased when measured within 60minutes post-game, remaining impaired for a further 48 hours. Whilst these data are useful, practitioners face difficulties when screening athletes during periods close to competition. Fatigue has been indicated as a mechanism to heighten injury risk in a range of sports due to changes in neuromuscular control (31). Therefore, practitioners may wish to assess their athletes' movement abilities under conditions of fatigue. Solely screening athletes in a nonfatigued state may not accurately identify those individuals whose movement mechanics deteriorate towards the end of competitions, affecting their relative risk of injury. Consequently, further research investigating the impact of fatigue on inter-limb differences is warranted using protocols which are practically viable.

Therefore, the aim of the present study was to examine the effects of a series of repeated sprints on unilateral jump performance and inter-limb asymmetries. It was hypothesized that 
Effects of Repeated Sprints on Asymmetries

heightened fatigue would result in decrements in jump performance and increased inter-limb asymmetries.

\section{METHODS}

\section{Experimental Approach to the Problem}

Subjects were required to attend on two separate occasions inclusive of a familiarization and a data collection session. After a standardised warm-up, subjects performed three maximal SLCMJ per leg, followed by four rounds of $6 \times 40 \mathrm{~m}$ sprints on a running track, that were interspersed with a SLCMJ on each leg to monitor the effects of repeated sprints on jump performance and inter-limb asymmetry. Subjects were instructed to refrain from any exercise for 48 hours before testing. This protocol provided the ability to evaluate the time-course of inter-limb asymmetry responses to the accumulated fatigue caused from repeated sprint training.

\section{Subjects}

Eighteen recreationally active males (age: $28.9 \pm 5.1$ years; height: $178.9 \pm 6 \mathrm{~cm}$; body mass: $83.4 \pm 13.7 \mathrm{~kg}$ ) were recruited and provided informed consent to take part in this study. All subjects regularly undertook physical activity including sporting activities and resistance training. Subjects were also required to have a minimum of two hours per week of lower body resistance training over the previous six months and be 'injury-free' for a minimum of three months at the time of testing. This study was approved by the London Sport Institute Research and Ethics Committee at Middlesex University, UK.

\section{Procedures}


Effects of Repeated Sprints on Asymmetries

Prior to testing. subjects performed a 10-minute standardised warm-up, consisting of a 5minute gentle jog, followed by five minutes of dynamic stretching (forward lunges, inchworms, lateral lunges, world's greatest stretch, and single leg glute bridge) and then three sub-maximal SLCMJ on each leg with increasing intensity of 50, 75 and $100 \%$ perceived maximal effort. A schematic of the study design is presented in Figure 1. Three maximal SLCMJ were recorded at baseline in order for test reliability to be computed in real time via the coefficient of variation (CV) with pre-set formulas in Microsoft Excel for each subject. Thirty seconds of rest was provided between each jump, beginning on the left leg and alternating legs for every jump. Subjects then sprinted $40 \mathrm{~m}$ six times, with 20 seconds of passive rest between each sprint. Subjects were permitted 120 seconds of rest following the final sprint, after which they performed one maximal effort SLCMJ on each leg, with 30 seconds of rest between each jump. Only one trial was conducted between sprints after noting that jump data was reliable during familiarization and at baseline. Following a further 120 seconds of rest, subjects carried out the sprint protocol in full again, repeating this until four total sets of repeated sprints were completed, recording one maximal effort SLCMJ per leg two minutes after the sixth sprint in each set (Figure 1). The distance and volume of sprints per set were chosen to ensure that an appropriate level of acute fatigue would be induced for the chosen sample (3).

\section{*** INSERT FIGURE 1 ABOUT HERE ***}

Single Leg Countermovement Jump (SLCMJ). Jump height was measured using a Just Jump mat (Probotics Inc., Huntsville, AL, USA) to the nearest $0.1 \mathrm{~cm}$. Subjects stood on one leg, looking straight ahead with hands placed on hips throughout the duration of the test. Subjects were instructed to jump as high as possible, beginning with a countermovement to a selfselected depth before accelerating vertically as fast as possible. The non-jumping leg was 
Effects of Repeated Sprints on Asymmetries

required to remain still beside the jumping leg throughout the jump. The jumping leg was required to remain extended during the flight phase of the jump. Take-off and landing occurred on the same leg, with the non-jumping leg remaining off the ground until the subjects had regained balance on the jumping leg. In such cases where these criteria were not met, the trial was considered void and was subsequently retaken after a 30-second rest.

$6 \times$ 40m Repeated Sprint Protocol. Single-beam electronic timing gates (Brower Timing Systems, Draper, UT, USA) were positioned on a running track $40 \mathrm{~m}$ apart. Sprints were initiated from a start line $0.3 \mathrm{~m}$ behind a timing gate, and subjects were instructed to sprint following a five-second countdown. Subjects sprinted to the opposite gate and were given verbal encouragement to sprint as fast as they could by researchers to ensure they produced their maximum effort. Subjects were instructed to be ready to perform the next sprint after 15 seconds of rest to ensure there were no delays and were given a five-second countdown to the start of each sprint. Rest periods were timed using a Gymboss Minimax stopwatch (Gymboss, St. Clair, MI, USA). The length of time taken to complete each sprint, as measured by the timing gates, was recorded to quantify the onset of fatigue.

\section{Calculating Asymmetry and Fatigue Index}

For calculations of SLCMJ asymmetry, the highest jump on each leg was used. The dominant limb was defined as the limb with the greatest jump height (7). Asymmetry values were calculated using the percentage difference method as suggested by Bishop et al. (6): $100 /($ maximum value $) *($ minimum value $) *-1+100$. The percentage decrement score equation by Glaister et al. (20) was used to establish each subjects' level of fatigue using the formula: 
Effects of Repeated Sprints on Asymmetries

$(100 *($ total sprint time/ideal sprint time) $)-100)$, where total sprint time $=$ sum of sprint times from all sprints and ideal sprint time = number of sprints multiplied by fastest sprint time.

\section{Statistical Analyses}

All sprint time and jump height data were recorded and inputted into Microsoft Excel ${ }^{\mathrm{TM}}$ to compute descriptive statistics (means and standard deviations (SD)). Subsequent statistical analysis was performed using SPSS (version 24.0, IBM Corporation, Armonk, NY, USA). Normality was assessed via the Shapiro-Wilk test. Within-session reliability was calculated at baseline using the coefficient of variation $(\mathrm{CV})$ : (SD/mean)*100, a two-way random intraclass correlation coefficient (ICC) with absolute agreement and standard error of measurement (SEM): SD*ل $(1-\mathrm{ICC}) . \mathrm{CV}$ values of $<10 \%$ were considered acceptable in accordance with Cormack et al. (14), and ICC values were interpreted in line with Koo and Li (25) where > 0.9 $=$ excellent, $0.75-0.9=$ good, $0.5-0.75=$ moderate, and $<0.5=$ poor.

A one-way repeated measures analysis of variance (ANOVA) with a Bonferroni confidence interval adjustment was used to examine within-set differences in sprint times, between-set differences in fatigue $\%$ and changes in mean jump height, to ensure that subjects fatigued from sprint one to sprint six in individual sets, and from the first sprint in set 1 to every subsequent set (See Equation 2). A one-way repeated measures ANOVA was also used to measure differences in jump height from the first SLCMJ to the last SLCMJ for each leg as a measure of fatigue, and to assess any changes in asymmetry from the first SLCMJ to each subsequent SLCMJ with statistical significance was set at $p<0.05$. Effect sizes (Cohen's $d$ ) were calculated as $\left(\mathrm{Mean}_{\mathrm{D}}-\mathrm{Mean}_{\mathrm{ND}} / \mathrm{SD}_{\text {pooled }}\right)$ to examine the magnitude of these differences and were interpreted where: $0.2-0.49=$ small, $0.5-0.79=$ medium and $>0.8=$ large (13). 
Effects of Repeated Sprints on Asymmetries

\title{
RESULTS
}

All data were normally distributed $(p>0.05)$, and baseline SLCMJ tests had acceptable between-trial consistency with mean $\mathrm{CV}$ values of 4.1 and $3.9 \%$ for left and right limbs, respectively. $\mathrm{ICC}$ scores were excellent $(\mathrm{left}=0.98$; right $=0.97)$ and $\mathrm{SEM}$ was $<0.01 \mathrm{~cm}$ on both limbs. Table 1 shows mean sprint performance and mean jump height for dominant (D) and non-dominant (ND) limbs across all five jump tests. Significant reductions in jump height $(p<0.05)$ were noted at all time points relative to baseline on both limbs. In addition, the ND limb also showed significant reductions in jump height after four sets of repeated sprints relative to sets one and two.

Figure 2 shows inter-limb asymmetry values at each stage of testing. Significant changes in asymmetry $(p<0.05)$ were noted after set three and four of the repeated sprint protocol relative to baseline testing; no other significant changes in asymmetry were noted. Figure 3 shows the sprint speed for the first and last sprints in each set. Significant differences $(p<0.05)$ in sprint speed were seen between the first and last sprint in each set; however, no significant differences between sets were noted. Figure 4 shows the mean fatigue index after each set of repeated sprints. Significant increases in fatigue $(p<0.01)$ were noted between sets one and two, three and four, sets two and three, and sets two and four.

\author{
*** INSERT TABLE 1 ABOUT HERE *** \\ *** INSERT FIGURES 2-4 ABOUT HERE ***
}


Effects of Repeated Sprints on Asymmetries

\section{DISCUSSION}

The aim of the present study was to examine the effects of a series of repeated sprints on unilateral jump performance and inter-limb asymmetries. Significant reductions in SLCMJ jump height were noted after each set of repeated sprints on both limbs. Inter-limb asymmetries increased after every set of sprints, with significant increases noted after three and four sets of sprints relative to baseline, indicating that asymmetry was heightened due to an accumulated fatigue effect.

Table 1 shows changes in SLCMJ jump height on both limbs and sprint speed at baseline and after each set of repeated sprints. For the SLCMJ, significant reductions in jump height were noted on the D limb after all sets, but only relative to the baseline set of jumps (ES $=-0.52$ to 0.74). The ND limb also showed the same trend; however, the final time point showed a significant reduction relative to sets one and two post-baseline jumps as well $(\mathrm{ES}=-0.54$ to 0.99). These results contrast to previous research, which has shown that jump height was not sensitive enough to detect true changes in a fatigued state $(10,18)$. Whilst challenging to explain conclusively, it must be acknowledged that the subjects in the present study were recreational athletes, where previous research has used college athletes (18) and elite academy soccer players (10). Thus, it is plausible that more experienced athletes have a greater ability to manipulate their jump strategy in order to achieve the same outcome. For mean sprint time, there was a significant increase in time taken to perform each set when compared to baseline. In addition, the second set of sprints (post-baseline) were significantly different from the first set and sets three and four showed significant increases in mean sprint time compared to all sets before them. Given the nature of the repeated task in question, it is no surprise that subjects performed subsequent sets significantly slower. This is in agreement with previous research, which has also showed significant reductions in repeated sprint performance (20). 
Effects of Repeated Sprints on Asymmetries

Figure 2 shows the inter-limb asymmetry values for jump height during the SLCMJ test at all time points. Inter-limb differences increased at each time point (baseline $=7.62 \%$, set $1=$ $9.82 \%$, set $2=9.95 \%$, set $3=13.25 \%$ and set $4=14.67 \%$ ), with significant differences seen between baseline and the third and fourth sets. Whilst the reduction in jump height was significant, there was no guarantee that asymmetry would also increase; thus, these data represent interesting findings. Furthermore, given the larger ES on the ND limb, it can be assumed that the increase in asymmetry was due to a greater reduction in jump performance on the ND limb. This concept has recently been acknowledged by Maloney (28) who suggested that asymmetries can be viewed as 'windows of opportunity' in respect to the ND limb, which is likely weaker and less capable of producing force rapidly. For example, given the scenario presented in the present study (i.e., with larger reductions in jump height on the ND limb), practitioners can use this data as a means to consider additional training for the ND limb. This concept was recently investigated by Brown et al. (11) who used supplementary strength and ballistic exercises for the weaker limb three times a week (for six weeks) in addition to the regular bilateral strength training programme for a sprint athlete. Inter-limb asymmetry reduced from 16 to $13 \%$ and improvements in maximal velocity $\left(5.86\right.$ to $\left.6.01 \mathrm{~m} \cdot \mathrm{s}^{-1}\right)$ and power $(18$ to $21 \mathrm{~W} \cdot \mathrm{kg}^{-1}$ ) were also noted. However, it should be acknowledged that this was a case study on a single athlete; thus, cannot be attributed to wider athlete populations. It is equally likely that unilateral training with the same absolute volume load could also correct asymmetries, given that the relative intensity, including the demands placed on stabilization will be greater on the ND leg. Thus, the ND leg is likely to experience a greater stimulus for adaptation (28).

Previous literature has suggested a wide range of thresholds lower than the $14.67 \%$ seen in the present study to be associated with reduced athletic performance. For example, Hart et al. (22) showed that strength asymmetries in peak force of $8 \%$ during the unilateral isometric squat were associated with reduced kicking accuracy when aiming at a target $20 \mathrm{~m}$ away. Maloney 
Effects of Repeated Sprints on Asymmetries

et al. (29) showed that jump height asymmetries from the unilateral drop jump (2.4-7.2\%) were strongly associated with slower change of direction speed performance during a task requiring multiple $90^{\circ}$ cuts. Recently, Bishop et al. (7) showed that jump height asymmetries (12.5\%) during the SLCMJ were associated with slower $5 \mathrm{~m}(r=0.49 ; p<0.05), 10 \mathrm{~m}(r=0.52 ; p<$ $0.05), 20 \mathrm{~m}$ sprints $(r=0.59 ; p<0.01)$ and unilateral jump performance $(r=-0.47$ to $-0.53 ; p$ $<0.05)$. In addition, previous research has also suggested that $10 \%$ may be a threshold for practitioners to consider during return to play when considering minimizing the risk of a second ACL injury (26). Therefore, in the present study, with asymmetries so close to that $10 \%$ threshold even after the first set of repeated sprints, practitioners may wish to monitor interlimb asymmetries as part of the recovery process. This has also been recently suggested by Bromley et al. (10) who showed how sensitive asymmetries were after academy soccer players had played a competitive match.

Figure 3 shows the mean sprint time for the first and last sprints during each set of repeated sprints. Significant increases in mean sprint time were seen between the first and last sprints in each set, suggesting that the dosage of $6 \mathrm{x} 40 \mathrm{~m}$ was sufficient to induce acute set to set changes in repeated sprint performance and between-limb jump height asymmetry from the SLCMJ. These findings are in contrast to Girard et al. (19) who undertook 5x5-second repeated sprints on a treadmill with 13 male recreational and racket-sport athletes where no meaningful changes in asymmetry were noted during the execution of the task. Thus, practitioners may confidently adopt this protocol to examine changes in jump performance and asymmetry under conditions of fatigue. In doing so, practitioners could consider that athletes who display marked increases in asymmetry could be targeted to increase their tolerance to fatigue as part of their conditioning strategy.

Figure 4 shows the fatigue index that accumulated as a result of more sets of repeated sprints conducted. Statistically significant levels of fatigue were observed between all sets with the 
Effects of Repeated Sprints on Asymmetries

exception of sets three and four. This further supports the notion that four sets of $6 \mathrm{x} 40 \mathrm{~m}$ sprints were an appropriate protocol to induce fatigue. This level of fatigue is in line with previous research by Glaister et al. (20), who demonstrated a fatigue of 5.13\% after one set of six $30 \mathrm{~m}$ sprints. As observed in the current study, fatigue did not significantly affect inter-limb asymmetries until the third set was completed, which corresponded to a fatigue index of 12.31\%. However, a significant reduction in jump height was observed after just the first set of sprints, which corresponded to a fatigue index of 5.74\%. Thus, it appears that jump height may be a more responsive metric than asymmetry when detecting true change in the present population. This is also likely due to the variable nature of asymmetry, noting that the SD of asymmetry was sometimes just as high as the mean (Figure 2). In contrast, the SD for jump height was approximately $30 \%$ at each time point. With that in mind, it is likely that responses to a training protocol such as repeated sprints will produce 'responders' and 'non-responders' in the context of asymmetry, suggesting that an individual approach to monitoring inter-limb differences may be required (5).

The present study was not without some limitations. Firstly, previous research has highlighted the importance of monitoring jump performance on a force platform where possible to report alterations in jump strategy, with jump height not always being sensitive enough to detect true change $(10,18)$. However, this is often not practically viable for testing in the field and the results of the present study have practical implications for a wider range of practitioners. Secondly, recent research has highlighted the individual nature of asymmetries (5); thus, these findings can only be attributed to this recreational athlete sample. Future research should aim to replicate this study with elite athletes using a more detailed analysis of mechanistic changes in jump strategy utilizing more sophisticated measurement tools. This will help provide a deeper understanding of the link between fatigue and inter-limb asymmetries within elite athletes. 
Effects of Repeated Sprints on Asymmetries

\section{PRACTICAL APPLICATIONS}

The findings of the present study show that if practitioners want to use a repeated sprint training session with the aim of acutely inducing fatigue, four sets of $6 \times 40 \mathrm{~m}$ sprints is an effective protocol. Given the frequent requirement for repeated sprinting for team sport athletes, this represents useful information for practitioners. In addition, given the variable nature of asymmetry (as represented by the high SD), practitioners should be mindful about concluding that this is a less sensitive metric than jump height alone. Instead, if practitioners use unilateral jump testing as part of the monitoring process, they are encouraged to assess inter-limb differences on an individual basis. This may help provide more accurate information about whether training loads should be manipulated or if targeted training interventions are required for individual athletes. 
Effects of Repeated Sprints on Asymmetries

\section{REFERENCES}

1. Bailey C, Sato K, Alexander R, Chiang C.-Y, and Stone M. Isometric force production symmetry and jumping performance in collegiate athletes. J Train 2: 1-5, 2013.

2. Bailey C, Sato K, Burnett A, and Stone M. Force production asymmetry in male and female athletes of differing strength levels. Int J Sports Physiol Perform 10: 504-508, 2015.

3. Balsom P, Seger Y, Sjodin B, and Ekblom B. Physiological responses to maximal intensity intermittent exercise. Eur J Appl Physiol Occup Physiol 65: 144-149, 1992.

4. Bell D, Sanfilipo J, Bikley N, and Heiderscheit B. Lean mass asymmetry influences force and power asymmetry during jumping in collegiate level athletes. J Strength Cond Res 28: 884-891, 2014.

5. Bishop C, Lake J, Loturco I, Papadopolous K, Turner A, and Read P. Interlimb asymmetries: The need for an individual approach to data analysis. J Strength Cond Res (Published ahead of print).

6. Bishop C, Read P, Lake J, Chavda S, and Turner A. Interlimb asymmetries: Understanding how to calculate differences from bilateral and unilateral tests. Strength Cond J 40: 1-6, 2018.

7. Bishop C, Read P, McCubbine J, and Turner A. Vertical and horizontal asymmetries are related to slower sprinting and jump performance in elite youth female soccer players. J Strength Cond Res (Published ahead of print).

8. Bishop C, Turner A, and Read, P. Training methods and considerations for practitioners to reduce interlimb asymmetries. Strength Cond J 40: 40-46, 2018.

9. Bishop C, Turner A, and Read P. Effects of inter-limb asymmetries on physical and sports performance: a systematic review. J Sports Sci 36: 1135-1144, 2018.

10. Bromley T, Turner A, Read P, Lake J, Maloney S, Chavda S, and Bishop C. Effects of a competitive soccer match on jump performance and interlimb asymmetries in elite academy soccer players. J Strength Cond Res (Published ahead of print).

11. Brown S, Feldman E, Cross M, Helms E, Marrier B, Samozino P, and Morin J-B. The potential for a targeted strength-training program to decrease asymmetry and increase performance: A proof of concept in sprinting. Int J Sports Physiol Perform 12: 13921395, 2017. 
12. Claudino J, Cronin J, Mezêncio B, McMaster D, McGuigan M, Tricoli V, and Serrão J. The countermovement jump to monitor neuromuscular status: A meta-analysis. J Sci Med Sport 20: 397-402, 2017.

13. Cohen J. Statistical power analysis for the behavioural sciences ( ${ }^{\text {nd }}$ Ed.). Hillsdale, New Jersey, Erlbaum.

14. Cormack S, Newton R, McGuigan M, and Doyle T. Reliability of measures obtained during single and repeated countermovement jumps. Int J Sports Physiol Perform 3: 131-144, 2008.

15. Dos'Santos T, Thomas C, Jones P, and Comfort P. Asymmetries in single and triple hop are not detrimental to change of direction speed. J Trainology 6: 35-41, 2017.

16. Evershed J, Burkett B, and Mellifont R. Musculoskeletal screening to detect asymmetry in swimming. Phys Ther Sport 15: 33-38, 2014.

17. Exell T, Irwin G, Gittoes M, and Kerwin D. Strength and performance asymmetry during maximal velocity sprint running. Scand J Med Sci Sports 27: 1273-1282, 2017.

18. Gathercole R, Sporer B, Stellingwerff T, and Sleivert G. Alternative countermovement jump analysis to quantify acute neuromuscular fatigue. Int J Sports Physiol Perform 10: 84-92, 2015.

19. Girard O, Brocherie F, Morin J-B, and Millet G. Lower limb mechanical asymmetry during repeated treadmill sprints. Human Move Sci 52: 203-214, 2017.

20. Glaister M, Howatson G, Pattison J, and McInnes G. The reliability and validity of fatigue measures during multiple-sprint work: An issue revisited. J Strength Cond Res 22: 1597-1601, 2008.

21. Gray J, Aginsky K, Derman W, Vaughan C, and Hodges P. Symmetry, not asymmetry, of abdominal muscle morphology is associated with low back pain in cricket fast bowlers. J Sci Med Sport 19: 222-226, 2017.

22. Hart N, Nimphius S, Spiteri T, and Newton R. Leg strength and lean mass symmetry influences kicking performance in Australian football. J Sports Sci Med 13: 157-165, 2014.

23. Hart N, Nimphius S, Weber J, Spiteri T, Rantalainen T, Dobbin M, and Newton R. Musculoskeletal asymmetry in football athletes: A product of limb function over time. Med Sci Sports Exerc 48: 1379-1387, 2016.

24. Hoffman J, Ratamess N, Klatt M, Faigenbaum A, and Kang J. (2007). Do bilateral power deficits influence direction-specific movement patterns? Res Sports Med 15: 125-132, 2007. 
25. Koo T, and Li M. A guideline of selecting and reporting intraclass correlation coefficients for reliability research. J Chiro Med 15: 155-163, 2016.

26. Kryitsis P, Bahr R, Landreau P, Miladi R, and Witvrouw E. Likelihood of ACL graft rupture: Not meeting six clinical discharge criteria before return to sport is associated with a four times greater risk of rupture. Brit J Sports Med 50: 946-951, 2016.

27. Lockie R, Callaghan S, Berry S, Cooke E, Jordan C, Luczo T, and Jeffriess M. Relationship between unilateral jumping ability and asymmetry on multidirectional speed in team-sport athletes. J Strength Cond Res 28: 3557-3566, 2014.

28. Maloney S. The relationship between asymmetry and athletic performance: A critical review. J Strength Cond Res (Published ahead of print).

29. Maloney S, Richards J, Nixon D, Harvey L, and Fletcher I. Do stiffness and asymmetries predict change of direction performance? J Sports Sci 35: 547-556, 2017.

30. Radzak K, Putnam A, Tamura K, Hetzler R, and Stickley C. Asymmetry between lower limbs during rested and fatigued state running gait in healthy individuals. Gait \& Posture 51: 268-274, 2017.

31. Read P, Oliver J, De Ste Croix M, Myer G, and Lloyd R. Consistency of field-based measures of neuromuscular control using force-plate diagnostics in elite youth male soccer players. J Strength Cond Res 30: 3304-3311, 2016.

32. Sannicandro I, Piccinno A, Rosa R, and De Pascalis S. Functional asymmetry in the lower limb professional soccer players. Brit J Sports Med 45: 370-372, 2011.

33. Schlitz M, Lehance C, Maquet D, Bury T, Crielaard J-M, and Croisier J-L. Explosive strength imbalances in professional basketball players. J Athl Train 44: 39-47, 2009.

34. Thorpe R, Atkinson G, Drust B, and Gregson W. Monitoring fatigue status in elite team-sport athletes: Implications for practice. Int J Sports Physiol Perform 12: 227 234, 2017. 


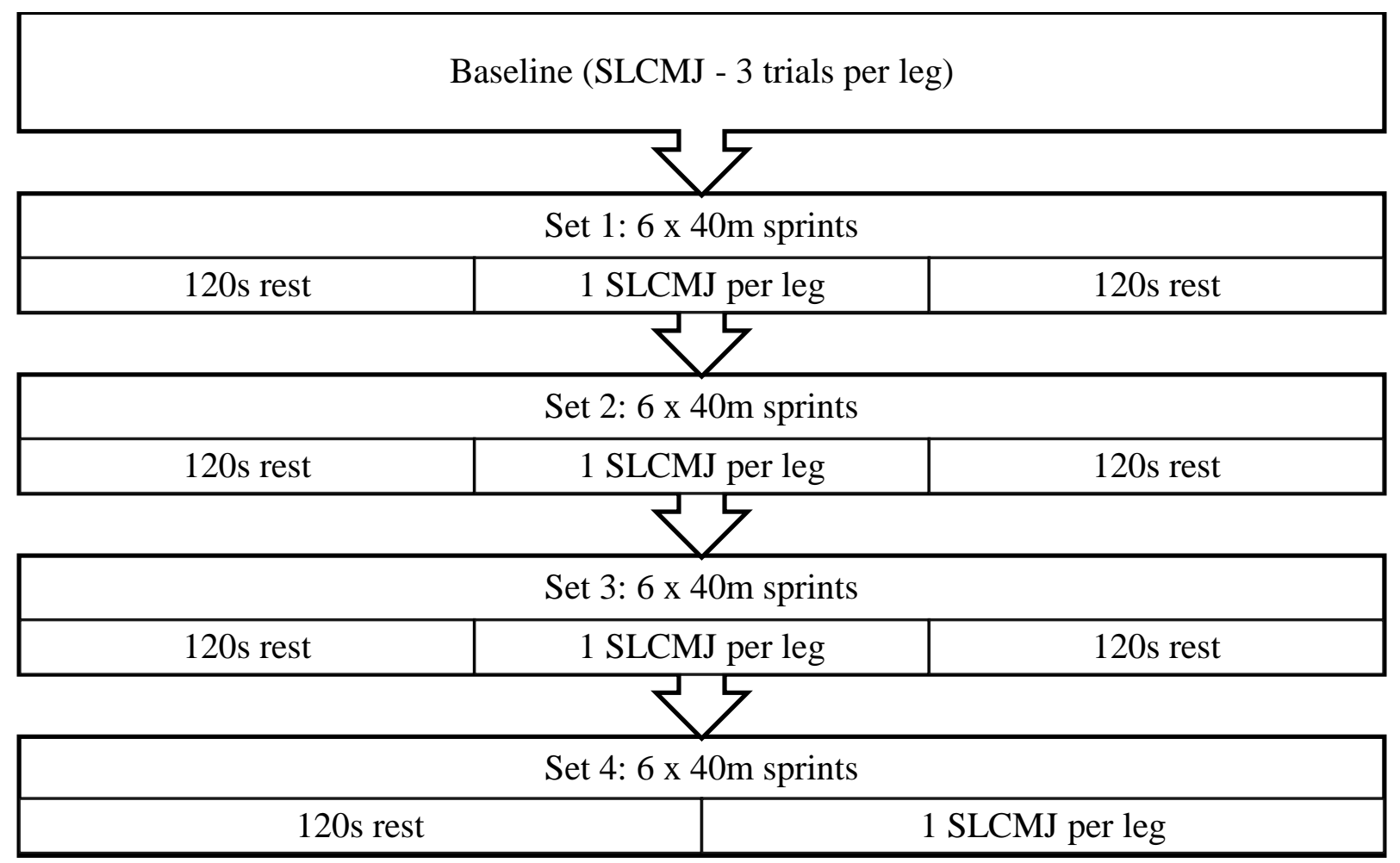

Figure 1: Schematic of study design (SLCMJ = single leg countermovement jump). 
Effects of Repeated Sprints on Asymmetries

Table 1: Mean jump height (in $\mathrm{cm}$ ) and sprint time (in seconds) \pm standard deviations (SD). Cohen's $d$ effect sizes are reported relative to baseline.

\begin{tabular}{|c|c|c|c|c|c|}
\hline Metric & Baseline & Set 1 & Set 2 & Set 3 & Set 4 \\
\hline JH-D & $15.73 \pm 4.47$ & $13.48 \pm 4.16^{\mathrm{a}}$ & $13.15 \pm 4.41^{\mathrm{a}}$ & $12.87 \pm 4.23^{\mathrm{a}}$ & $12.58 \pm 4.02^{\mathrm{a}}$ \\
\hline Effect size & & -0.52 & -0.58 & -0.66 & -0.74 \\
\hline JH-ND & $14.48 \pm 4.08$ & $12.23 \pm 4.25^{\mathrm{a}}$ & $11.72 \pm 3.72^{\mathrm{a}}$ & $11.11 \pm 3.55^{\mathrm{a}}$ & $10.71 \pm 3.53^{\mathrm{abc}}$ \\
\hline Effect size & & -0.54 & -0.71 & -0.88 & -0.99 \\
\hline Sprint time & $5.76 \pm 0.48$ & $6.08 \pm 0.46^{\mathrm{a}}$ & $6.26 \pm 0.48^{\mathrm{ab}}$ & $6.46 \pm 0.46^{\mathrm{abc}}$ & $6.52 \pm 0.49^{\mathrm{abc}}$ \\
\hline Effect size & & 0.69 & 1.05 & 1.48 & 1.58 \\
\hline
\end{tabular}

$\mathrm{JH}=$ jump height; $\mathrm{D}=$ dominant; $\mathrm{ND}=$ non-dominant; ${ }^{\mathrm{a}}$ significantly different to baseline $(p<0.05)$; ${ }^{\mathrm{b}}$ significantly different to set $1(p<$ $0.05) ;{ }^{c}$ significantly different to set $2(p<0.05)$. 
Effects of Repeated Sprints on Asymmetries

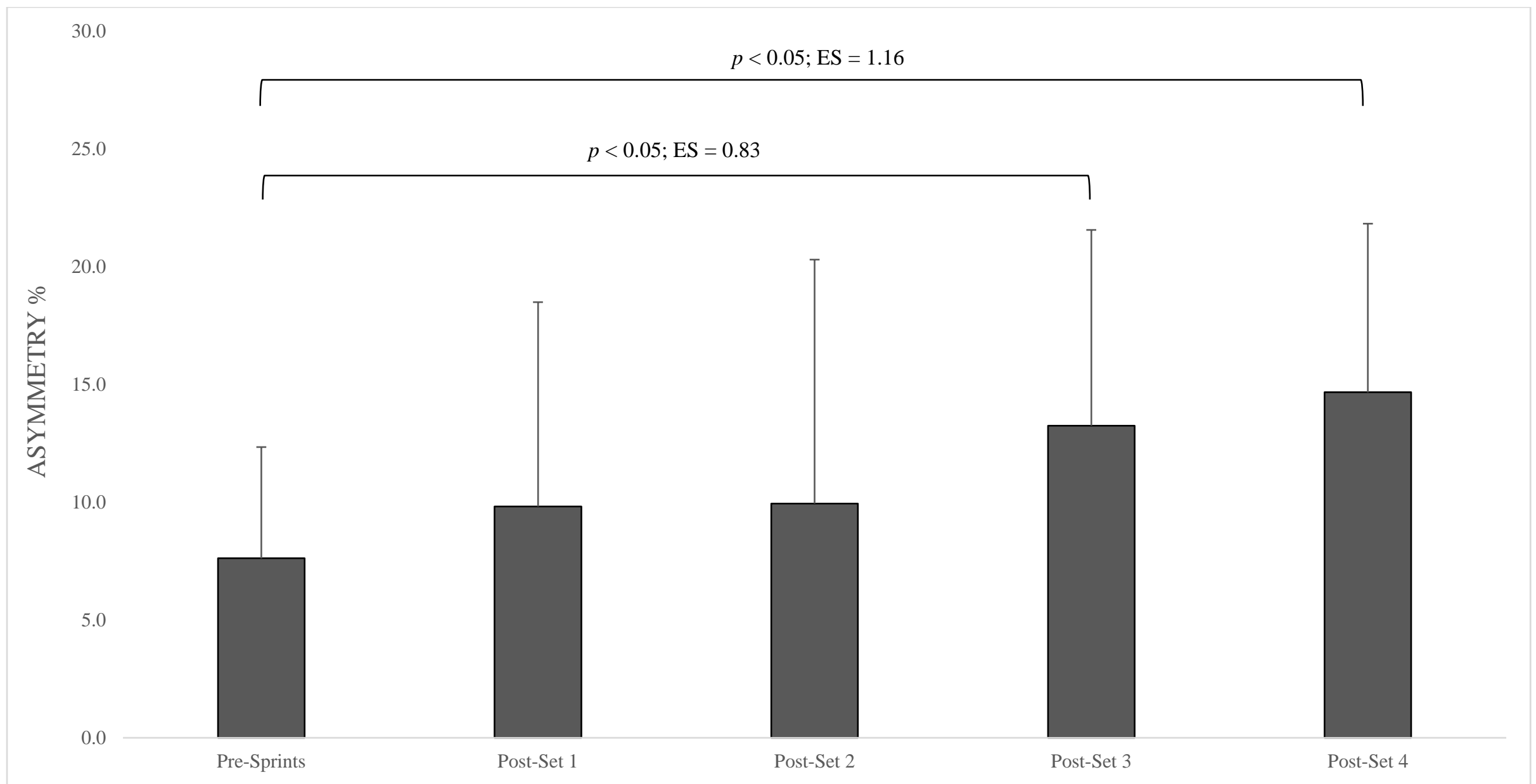

Figure 2: Inter-limb asymmetry values from the single leg countermovement jump test pre-sprints and after one, two, three and four sets of repeated sprints $(\mathrm{ES}=$ effect size). 
Effects of Repeated Sprints on Asymmetries



Figure 3: Sprint times for sprint 1 and sprint 6 in Sets 1-4. Significant $(p<0.05)$ difference between sprint 1 and all subsequent sprints in every set $(\mathrm{ES}=$ effect size $)$. 
Effects of Repeated Sprints on Asymmetries

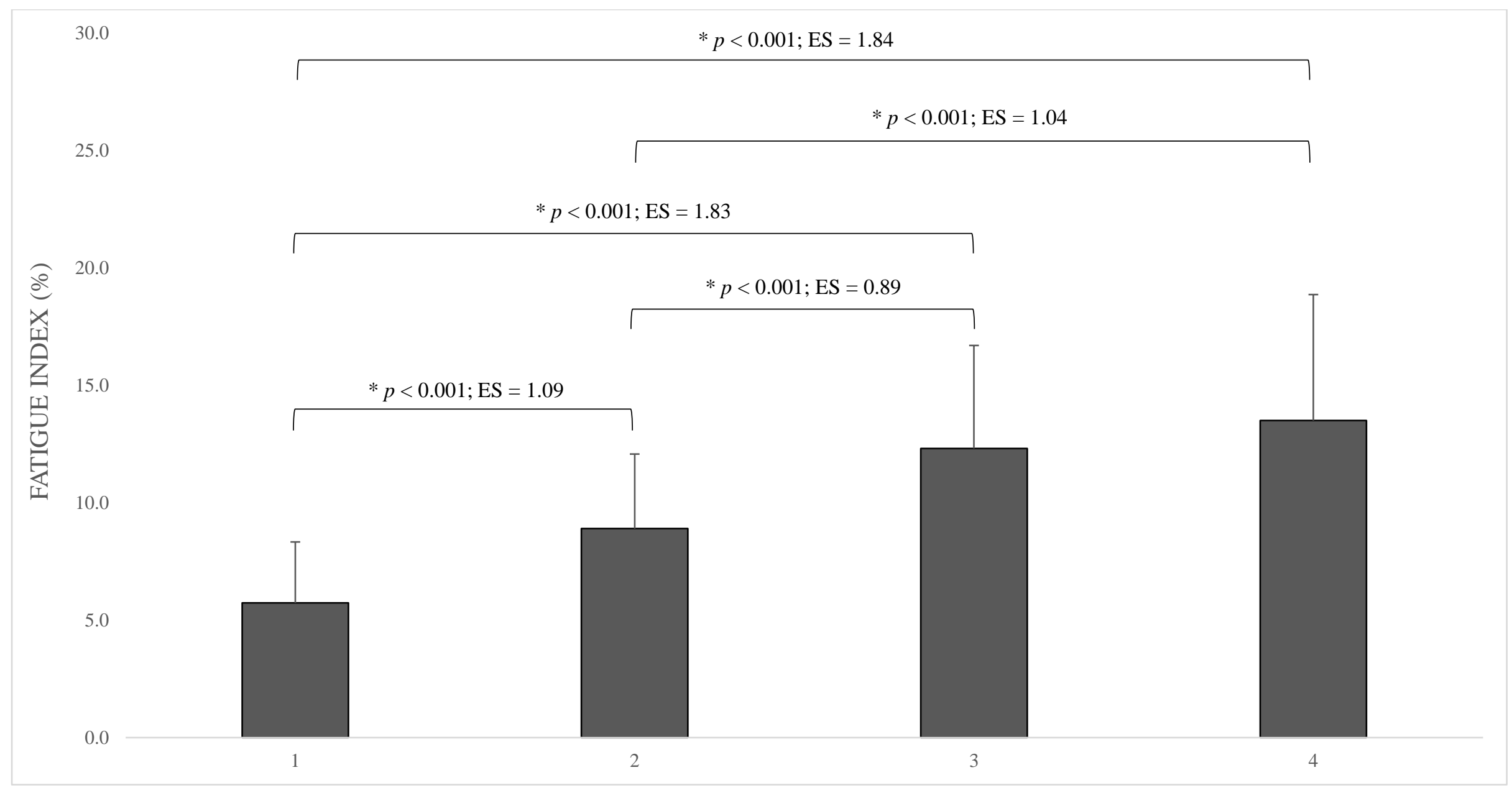

Figure 4: Fatigue index (expressed as a percentage) and standard deviations (error bars) after each set of repeated sprints $($ ES $=$ effect size). 